\title{
Immunohistochemical Identification of AQP1 in Livers of the High-fat Diet Rats
}

\author{
Identificación Inmunohistoquímica de AQP1 en Hígados \\ de Ratas con Dieta de Alto Contenido de Grasa
}

Jinbao Wang ${ }^{1}$; Pei Wang ${ }^{2} \&$ Wen Yang ${ }^{1}$

WANG, J.; WANG, P. \& YANG, W. Immunohistochemical identification of AQP1 in livers of the high-fat diet rats. Int. J. Morphol., 37(3):815-820, 2019.

SUMMARY: One of the key functions of the hepatobiliary system is bile formation. Aquaporins (AQPs) are likely to play a role in water transport that is essential for appropriate hepatobiliary tract function. The increasing prevalence of fatty liver parallels the rise of obesity and its complications over the past several decades. In this paper, general morphology observation, histopathology and AQP1 immunohistochemical expression were observed in livers of the high-fat diet (HFD) rats. For the liver of HFD rats, immunolight microscopy revealed weak labeling of AQP1 on the surface of central veins and liver sinusoid compared with the normal diet (ND) rats. It was suggested that bile secreted by the liver of HFD rats was maybe abnormal, thereby causing abnormalities in the composition and secretion of bile. However, the deeper understanding of mechanisms involved to the fatty liver is still unclear, in particular AQPs in the liver of obesity, additional studies would be required to study the signalling cascades involved in these processes.

KEY WORDS: Obesity; Liver; AQP1; Fatty liver.

\section{INTRODUCTION}

One of the key functions of the hepatobiliary system is bile formation. In humans, $0.5-1.0$ liters of bile are secreted by the liver. Bile consists of more than $98 \%$ water (Ma et al., 2001). Therefore, aquaporins (AQPs) are likely to play a role in water transport that is essential for appropriate hepatobiliary tract function. Cells from the hepatobiliary tract including hepatocytes, cholangiocytes, gallbladder epithelial cells, and endothelial cells from blood vessels have indeed been shown to express various AQPs that could account for transcellular water transport (Masyuk \& LaRusso, 2006; Portincasa \& Calamita, 2012; Gregoire et al., 2015).

As is well known, AQPs are small and represent a family of transmembrane proteins, which allow the transcellular water transport driven by osmotic forces, and namely facilitate regulated water transport. In mammals, thirteen AQPs e.g. AQP0-AQP12 have been detected so far, mainly classical AQPs, essentially permeable to water (AQP02, AQP4-6, AQP8) (Ishibashi et al., 2014). It was reported that in rats, secretin induces, via a cAMP-dependent pathway, exocytotic insertion of AQP1 in the apical membrane of cholangiocytes and facilitates the secretion of water into the biliary lumen (Tietz et al., 2003; Splinter et al., 2003).
Obesity is the state in which surplus energy accumulates as fat as a result of excessive energy intake and low energy expenditure due to lack of exercise. As a serious health issue particularly in this century, obesity increases the risk of developing diabetes, fatty liver, hypertension, hyperlipidemia, metabolic syndrome, coronary heart disease, and stroke (Wilson et al., 2002; Meshkani \& Adeli, 2009). And it was suggested that excess lipid accumulation may lead to lipotoxicity and may be the major driver of organ dysfunction (Declèves \& Sharma, 2015).

AQP1 is expressed at several sites that are potentially involved in dietary fat processing, including intrahepatic cholangiocytes (bile secretion) (Ma et al.). Secretin-regulated trafficking of AQP1 in cholangiocytes has been proposed to occur in bile secretion (Marinelli et al., 1999). For the highfat nutritional obesity, one of the most important clinical manifestations was fatty liver. However, hepatic expression of AQP1 in the fatty liver is still unclear, is there upregulation or down- regulation of protein level? Therefore, we aimed to copy the mode of high-fat nutritional obesity, and determine AQP1 expression and related functions in the obesity, thereby providing potential cross-talk between the bile secretion and the fatty liver disease.

\footnotetext{
${ }^{1}$ Laboratory Animal Center, Ningxia Medical University and Ningxia Hui Autonomous Region, Yinchuan, 750004 Ningxia, China.

${ }^{2}$ School of Basic Medicine, Ningxia Medical University, Yinchuan, 750004 Ningxia, China.
} 


\section{MATERIAL AND METHOD}

All experimental procedures and protocols in the study were approved by the Animal Ethics Committee of the Ningxia Hui Autonomous Region, China.

Sprague Dawley (SD) rats were obtained from the Laboratory Animal Center, Ningxia Medical University and Ningxia Hui Autonomous Region, and bred in the Laboratory Centre. To avoid the impact of sex on this experiment, healthy and male SD aged about 8 weeks and weighting about $240 \mathrm{~g}$ were used at the beginning of the experiment $(n=60)$. All animals were bred in-house and had free access to clean water and rat chow for the duration of the study. After 1 week of adaptive feeding, the rats were randomly divided into two groups, normal diet (ND, $\mathrm{n}=30$ ) and high-fat diet (HFD, $n=30$ ), respectively. ND was fed with normal rat chow, the fat of which accounted for $10 \%$ of the total calories. Meanwhile, HFD was fed with High-fat Diet, where the fat accounted for $66 \%$ of the total calories (Carbohydrate: 15.48 $\%$; Protein: $18.08 \%$; Fat: $66.43 \%$, lard-saturated fatty acid mainly). Animals were housed in steel cages in a controlled temperature room at $23 \pm 2{ }^{\circ} \mathrm{C}$, exposed to a daily 12-hour light-dark cycle (lights on at 07:00 a.m. and off at 07:00 p.m.). Hereditary obesity (ND) and obesity-resistant (HFD) were artificially eliminated during the study. After half-year feeding, the livers were studied by general morphological observation, then liver samples were collected and immediately fixed with $4 \%$ paraformaldehyde solution (PH7.4), and shortly afterward, blocks were cut from various parts of the kidneys. To conduct histologic studies, sections were stained with hematoxylin and eosin (HE).

For immunohistological studies, specimens were dehydrated through a graded series of alcohol, cleared and embedded in paraffin wax, and sectioned at $5 \mathrm{~mm}$ used for immunohistochemical staining. The sections were dewaxed and rehydrated, an antigen retrieval procedure was performed to unmask antigens by treating the samples three times in a microwave oven at medium-high fire for $5 \mathrm{~min}$ each time in $10 \mathrm{mM}$ citrate buffer, $\mathrm{pH}$ 6. After cooling to room temperature, the sections were treated with $3 \%$ hydrogen peroxide $\left(\mathrm{H}_{2} \mathrm{O}_{2}\right)$ in distilled water for $30 \mathrm{~min}$, then washed three times with PBS for 10 min each. The sections were incubated in blocking reagent for $1 \mathrm{~h}$ and incubated overnight at $4{ }^{\circ} \mathrm{C}$ with anti-AQP1 diluted 1:200 (Boster, Wuhan, China). The primary antibody was diluted in $10 \mathrm{mM}$ PBS supplemented with $0.1 \%$ BSA and $0.3 \%$ Triton X-100 [10 mM PBS (7mM Na2HPO4, 3mM NaH2PO4, $150 \mathrm{mM} \mathrm{NaCl}$, $\mathrm{pH}$ 7.4)]. Subsequently, the sections were rinsed with 10 $\mathrm{mM}$ PBS for $3 \times 10 \mathrm{~min}$ and incubated with biotinylated antirabbit IgG (ZSGB-BIO, Beijing, China, dilution: 1:200) secondary antibody for $2 \mathrm{~h}$ at room temperature. Then, the sections were rinsed with $10 \mathrm{mM}$ PBS for $3 \times 10 \mathrm{~min}$ and incubated with horseradish peroxidase streptavidin (ZSGBBIO, Beijing, China, dilution: 1:200) tertiary antibody for 2 $\mathrm{h}$ at room temperature followed by coloration with diaminobenzidine (DAB; ZSGB-BIO, Beijing, China) for 30 s to visualize positive reaction. Counterstaining was with hematoxylin staining. The sections were carried out using bright microscopy (NI-V, Nikon, Japan).

\section{RESULTS}

General morphological observation. General morphological observation of body shape and liver at ND and HFD rats were showed in Figure 1, and the different lobes of liver in rats also were observed, e.g. 1, right lateral lobe; 2 , right medial lobe; 3 , left medial lobe; 4, left lateral lobe (Popseko et al., 1992). Macroscopically, the liver of ND rats was reddish-brown, with smooth surface, sharp edges, soft and elastic. However, compared with ND rats, the liver of HFD rats was brownish yellow, and the swollen capsule was tight. Besides, the cut surface was greasy (Fig. 1).

Histologic features. The structure of the liver of ND and HFD rats were examined in the sections stained with HE (Fig. 2). Under light microscope, the structure of hepatic lobule of ND rats was normal, which were in close contact along most of their length, making it difficult to establish the exact limits between different lobules. The liver sinusoids (vas sinusoideum) ran radially and converged in the center of the lobule to form the central vein (venae centrales hepatis), this vessel has thin walls consisting of only endothelial cells supported by a sparse population of collagen fibers. Moreover, the structure of hepatocytes was clear, which of the cytoplasm was abundant and the nucleus located at the center (Fig. 2A-D). However, for the liver of HFD rats, the hepatic cords appear to were be arranged disorderly. Hepatocytes were ballooned, which of the cytoplasm was loosening and nucleus was squeezed to the edge. There were vacuoles of different sizes in the cytoplasm, termed MalloryDenk bodies. In addition, significant inflammatory cell infiltration occurred in the portal area and central vein and hepatocyte necrosis were observed. Lipid-forming hepatocytes accounted for more than $50 \%$ of hepatic lobules (Fig. 2E-H).

Protein examination. A deeper understanding of AQP1 expression would provide useful information for liver pathophysiology. Pronounced AQP1 immunohistochemical expression was detected on the surface of central vein and liver sinusoid of ND rats, presumably reacting with the 


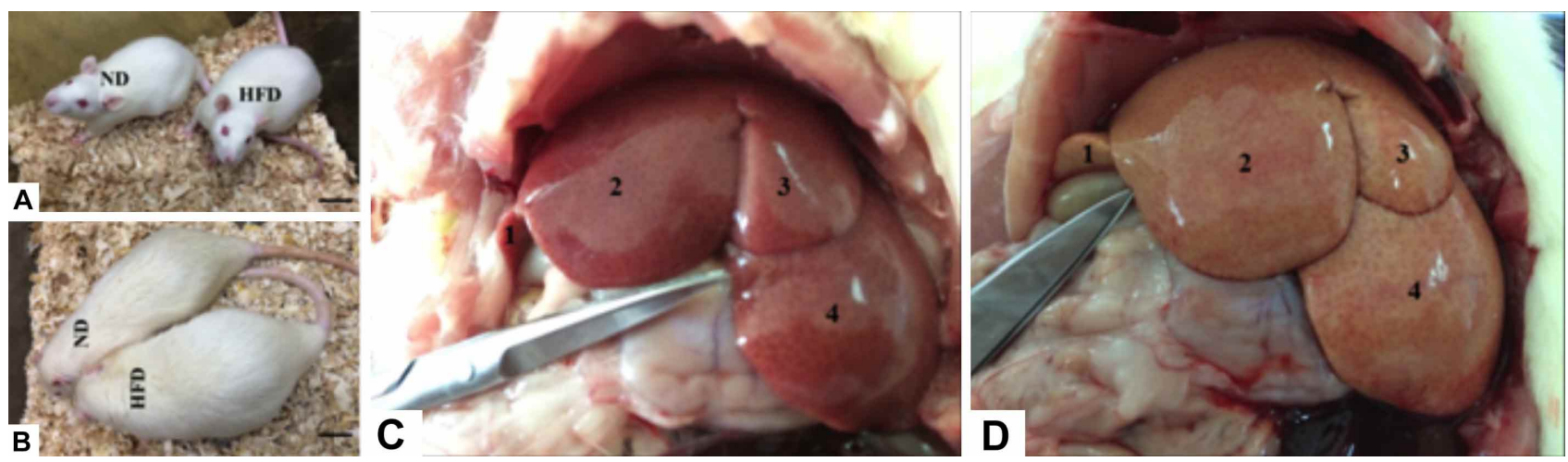

Fig. 1. General morphological observation of body shape and liver at ND and HFD rats. (A) SD rat aged 8 weeks; (B) SD rat aged 32 weeks; (C\&D) Morphological observation of liver at ND and HFD rats, respectively (ND, Normal Diet; HFD, High-fat Diet; 1, right lateral lobe; 2 , right medial lobe; 3 , left medial lobe; 4 , left lateral lobe.
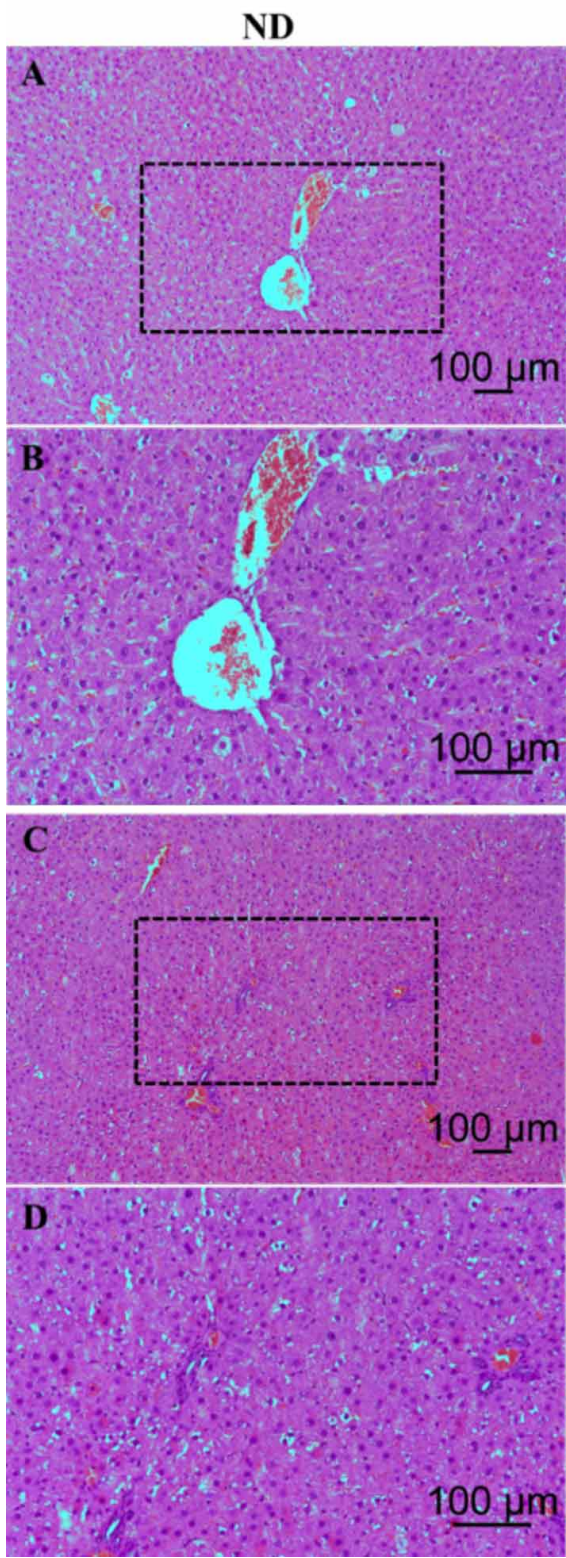

HFD
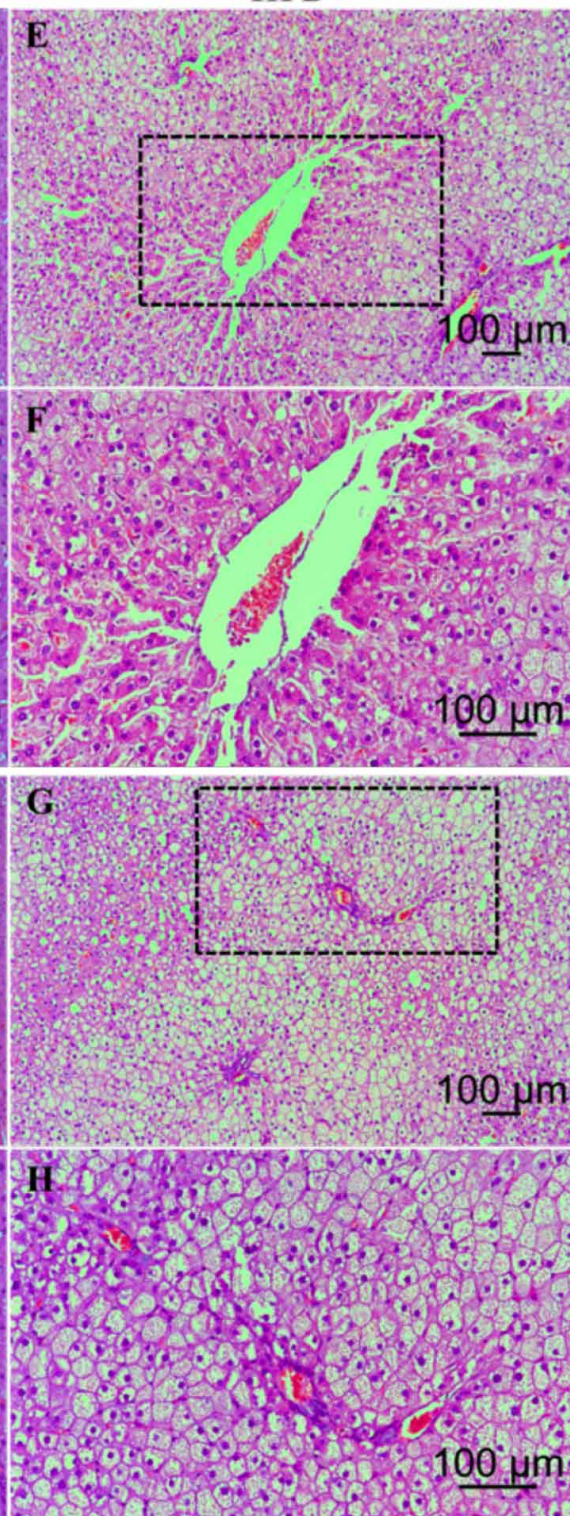

endothelial cells lining the central vein and liver sinusoid. But hepatocytes were negative for antiAQP1 reactivity (Fig. 3A-D). However, for the liver of HFD rats, immunolight microscopy revealed weak labeling of AQP1 on the surface of central veins and liver sinusoid compared with ND rats. And hepatocytes of HFD rats were also negative reactivity for antiAQP1 (Fig. 3E-H).
Fig. 2. Histologic features of livers at ND (A-D) and HFD (E-H) rats in HE staining on paraffin section. $\mathrm{B} / \mathrm{F}$ and $\mathrm{D} /$ $\mathrm{H}$ were the higher magnification images of the black box region in $\mathrm{A} / \mathrm{E}$ and $\mathrm{C} / \mathrm{G}$, respectively. 


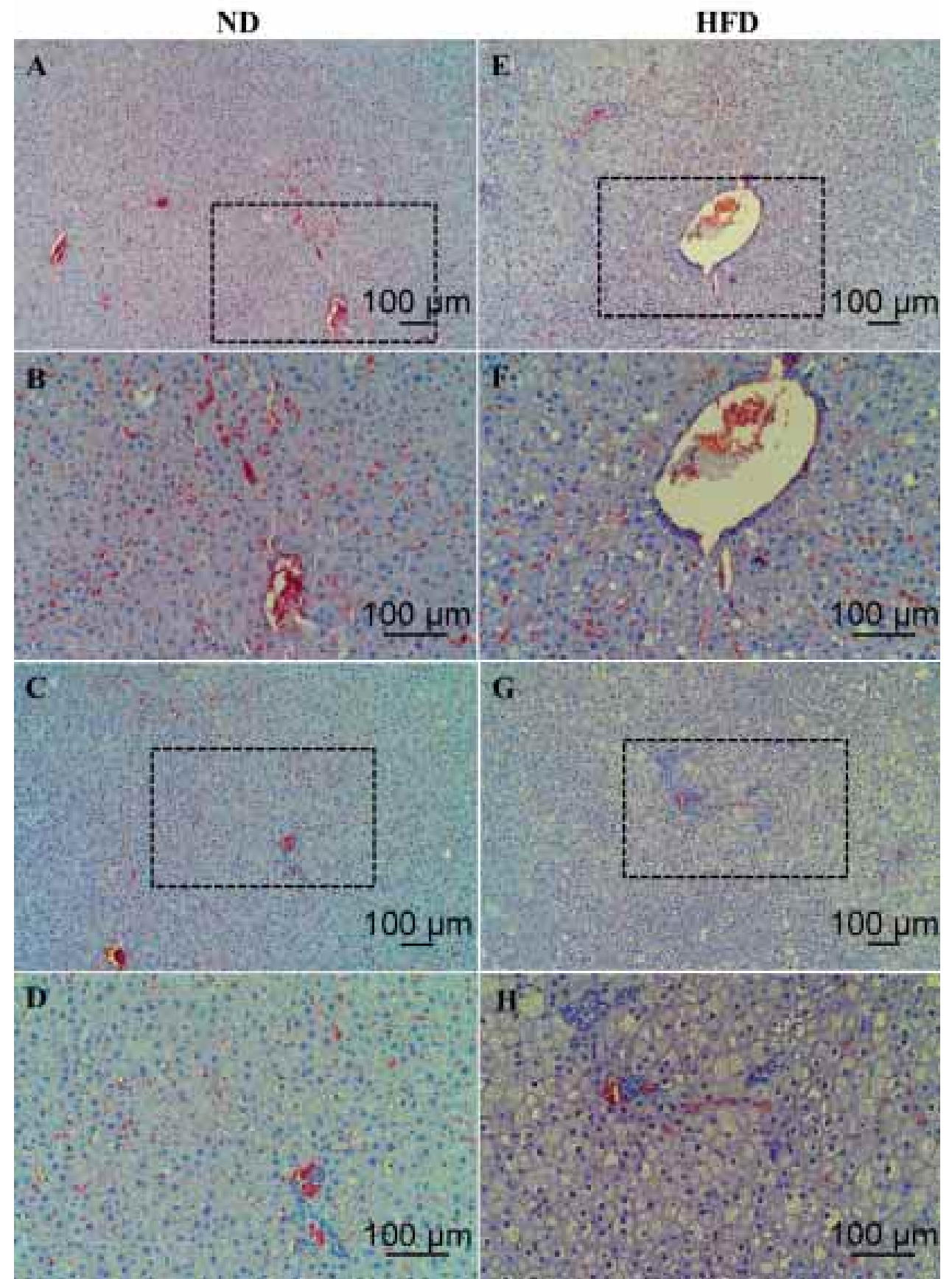

Fig. 3. Immunohistochemical expression of AQP1 in the livers tissue section from ND (A-D) and $\mathrm{HFD}(\mathrm{E}-\mathrm{H})$ rats. $\mathrm{B} / \mathrm{F}$ and $\mathrm{D} / \mathrm{H}$ were the higher magnification images of the black box region in $\mathrm{A} / \mathrm{E}$ and $\mathrm{C} / \mathrm{G}$, respectively.

\section{DISCUSSION}

The increasing prevalence of fatty liver parallels the rise of obesity and its complications over the past several decades, here referring in particular to primary nonalcoholic fatty liver disease (NAFLD), namely fatty liver is caused by over nutrition. The presentation of the disease ranges from what can be considered as "silent liver 
disease", or fatty steatosis. NAFLD is characterized by inflammation, cellular injury and fibrosis. And the cellular injury in NAFLD is characterized by ballooned hepatocytes, which frequently contain Mallory-Denk bodies, including $>5 \%$ of hepatocytes infiltrated with fat in individuals with no history of alcohol abuse (Kleiner \& Brunt, 2012; Milic'\& Stimac, 2012). In this study, as longterm high-fat diet, whether it is general morphology observation or histopathology, clinical symptoms of fatty liver were both observed in the different lobes of liver in HFD rats. Here hepatic injury was involved in insulin resistance, lipotoxicity, mitochondrial dysfunction, oxidative stress and endoplasmic reticulum stress and so on (Koppe, 2014).

NAFLD is strongly linked to obesity, with a reported prevalence as high as $80 \%$ in obese patients and only 16 $\%$ in individuals with a normal body mass index and without metabolic risk factors (Williams et al., 2011). Immunocytochemistry showed expression of AQP1 water channels at sites involved in dietary fat processing, including intrahepatic cholangiocytes, gallbladder, pancreatic microvascular endothelium and intestinal lacteals (Ma et al.). Moreover, it was reported that AQP1 was expressed in endothelial cells from venules and sinoids, and endothelial AQP1 expression probably facilitates water transport from plasma to bile (Yokomori et al., 2011; Gregoire et al.). Compared with ND rats in this study, immunolight microscopy revealed weak AQP1 expression in endothelial cells of central veins/venules and liver sinusoid in the liver of HFD rats. It was suggested that bile secreted by the liver of HFD rats was maybe abnormal, thereby causing abnormalities in the composition and secretion of bile.

AMP-activated protein kinase (AMPK), a serine/ threonine kinase, acts as an energy sensor in the homeostasis of carbohydrate and lipid metabolisms (Kahn et al., 2005). Recently, it has emerged as being involved in mediating endocrine regulation of metabolic fluxes in the liver (Hasenour et al., 2013). AICAR, as an AMPK activator, has been shown to significantly decreased AQP1, AQP9, and AQP11 mRNA levels in primary culture of murine hepatocytes (Gregoire et al.). And the classical antidiuretic hormone AVP does not regulate the expression of AQP1 (Terris et al., 1996). Thereby, there was a hypothesis that the down-regulation effect of AICAR on AQP1 expression levels in the liver of HFD rats was maybe a new finding. Of course, we needed to exclude that possibility, namely species-specific regulation of AQP1 was differentiated in the mouse, rat and human and so on.

In our study, we established the model of the high- fat nutritional obesity of rat, and hereditary obesity and obesity-resistant were artificially eliminated during the study. To some extent, general morphology observation, histopathology and weak AQP1 expression in the liver of HFD rats provided evidences for fatty liver disease, particularly in protein examination of AQP1. It was that AQP1 was potentially involved in bile formation processing. As mentioned, AQP1 was regulated by AMPK mediating AICAR. It also was reported that a gut peptide stimulating appetite, ghrelin decreased AMPK activation and increased metabolic enzymes transcript levels in the liver of rats (Kola et al., 2005). Therefore, additional studies on AMPK and ghrelin in the liver of HFD rats would be required to study the signaling cascades involved in these processes, as well as the physiological relevance.

\section{ACKNOWLEDGMENTS}

We thank Lirong Zhou and Yanping Zhu (Undergraduates) for establishment the mode of high-fat nutritional obesity, and Hongbing Li for assistance with sample collection in this study. This work was supported by Higher Education Scientific Research Project of Ningxia (NGY2017091) and the Project of Ningxia Medical University (XT2017006), China.

WANG, J.; WANG, P. \& YANG, W. Identificación inmunohistoquímica de AQP1 en hígados de ratas con dieta de alto contenido de grasa. Int. J. Morphol., 37(3):815-820, 2019,

RESUMEN: Una de las funciones clave del sistema hepatobiliar es la formación de bilis. Es probable que las acuaporinas (AQP) desempeñen un papel en el transporte de agua que es esencial para la función apropiada del tracto hepatobiliar. En las últimas décadas, la creciente prevalencia de hígado graso es paralela al aumento de la obesidad y sus complicaciones. En este trabajo, se identificaron características morfológicas generales, histopatología y expresión inmunohistoquímica de AQP1 en hígados de ratas con dieta rica en grasas (DRG). En el hígado de ratas con DRG, la expresión inmunohistoquímica determinó un marcaje débil de AQP1 en la superficie de las venas centrales y del sinusoide hepático en comparación con las ratas de dieta normal (DN). Se sugirió que la bilis secretada por el hígado de ratas con DRG era tal vez anormal, lo que causaba anomalías en la composición y secreción de la bilis. Sin embargo, se necesita un conocimiento mayor de los mecanismos involucrados en el hígado graso, en particular de las AQP y se requieren estudios adicionales para determinar las cascadas de señalización involucradas en estos procesos.

PALABRAS CLAVE: Obesidad; Hígado; AQP1; Hígado graso. 


\section{REFERENCES}

Declèves, A. E. \& Sharma, K. Obesity and kidney disease: differential effects of obesity on adipose tissue and kidney inflammation and fibrosis. Curr. Opin. Nephrol. Hypertens., 24(1):28-36, 2015.

Gregoire, F.; Lucidi, V.; Zerrad-Saadi, A.; Virreira, M.; Bolaky, N.; Delforge, V.; Lemmers, A.; Donckier, V.; Devière, J.; Demetter, P.; et al. Analysis of aquaporin expression in liver with a focus on hepatocytes. Histochem. Cell Biol., 144(4):347-63, 2015.

Hasenour, C. M.; Berglund, E. D. \& Wasserman, D. H. Emerging role of AMP-activated protein kinase in endocrine control of metabolism in the liver. Mol. Cell. Endocrinol., 366(2):152-62, 2013.

Ishibashi, K.; Tanaka, Y. \& Morishita, Y. The role of mammalian superaquaporins inside the cell. Biochim. Biophys. Acta, 1840(5):150712, 2014.

Kahn, B. B.; Alquier, T.; Carling, D. \& Hardie, D. G. AMP-activated protein kinase: ancient energy gauge provides clues to modern understanding of metabolism. Cell Metab., 1(1):15-25, 2005.

Kleiner, D. E. \& Brunt, E. M. Nonalcoholic fatty liver disease: pathologic patterns and biopsy evaluation in clinical research. Semin. Liver Dis., 32(1):3-13, 2012

Kola, B.; Hubina, E.; Tucci, S. A.; Kirkham, T. C.; Garcia, E. A.; Mitchell, S. E.; Williams, L. M.; Hawley, S. A.; Hardie, D. G.; Grossman, A. B.; et al. Cannabinoids and ghrelin have both central and peripheral metabolic and cardiac effects via AMP-activated protein kinase. J. Biol. Chem., 280(26):25196-201, 2005.

Koppe, S. W. Obesity and the liver: nonalcoholic fatty liver disease. Transl. Res., 164(4):312-22, 2014.

Ma, T.; Jayaraman, S.; Wang, K. S.; Song, Y.; Yang, B.; Li, J.; Bastidas, J. A. \& Verkman, A. S. Defective dietary fat processing in transgenic mice lacking aquaporin-1 water channels. Am. J. Physiol. Cell Physiol., 280(1):C126-34, 2001.

Marinelli, R. A.; Tietz, P. S.; Pham, L. D.; Rueckert, L.; Agre, P. \& LaRusso, N. F. Secretin induces the apical insertion of aquaporin-1 water channels in rat cholangiocytes. Am. J. Physiol., 276(1):G280-6, 1999.

Masyuk, A. I. \& LaRusso, N. F. Aquaporins in the hepatobiliary system. Hepatology, 43(2 Suppl. 1):S75-81, 2006.

Meshkani, R. \& Adeli, K. Hepatic insulin resistance, metabolic syndrome and cardiovascular disease. Clin. Biochem., 42(13-14):1331-46, 2009.

Milic, S. \& Stimac, D. Nonalcoholic fatty liver disease/steatohepatitis: epidemiology, pathogenesis, clinical presentation and treatment. Dig. Dis., 30(2):158-62, 2012.

Popseko, P.; Rajtová, V.; Horák, J. \& Timcáková, I. A Colour Atlas of the Anatomy of Small Laboratory Animals: Rat, Mouse, Golden Hamster. London, Wolfe Publishing, 1992.

Portincasa, P. \& Calamita, G. Water channel proteins in bile formation and flow in health and disease: when immiscible becomes miscible. Mol. Aspects Med., 33(5-6):651-64, 2012.

Terris, J.; Ecelbarger, C. A.; Nielsen, S. \& Knepper, M. A. Long-term regulation of four renal aquaporins in rats. Am. J. Physiol., 271(2 Pt. 2):F414-22, 1996

Tietz, P. S.; Marinelli, R. A.; Chen, X. M.; Huang, B.; Cohn, J.; Kole, J.; McNiven, M. A.; Alper, S. \& LaRusso, N. F. Agonist-induced coordinated trafficking of functionally related transport proteins for water and ions in cholangiocytes. J. Biol. Chem., 278(22):20413-9, 2003.

Williams, C. D.; Stengel, J.; Asike, M. I.; Torres, D. M.; Shaw, J.; Contreras, M.; Landt, C. L. \& Harrison, S. A. Prevalence of nonalcoholic fatty liver disease and nonalcoholic steatohepatitis among a largely middleaged population utilizing ultrasound and liver biopsy: a prospective study. Gastroenterology, 140(1):124-31, 2011.

Wilson, P. W.; D'Agostino, R. B.; Sullivan, L.; Parise, H. \& Kannel, W. B. Overweight and obesity as determinants of cardiovascular risk: the Framingham experience. Arch. Intern. Med., 162(16):1867-72, 2002.
Yokomori, H.; Oda, M.; Yoshimura, K.; Kaneko, F. \& Hibi, T. Aquaporin1 associated with hepatic arterial capillary proliferation on hepatic sinusoid in human cirrhotic liver. Liver Int., 31(10):1554-64, 2011.

\author{
Corresponding author: \\ Jinbao Wang \\ Laboratory Animal Center \\ Ningxia Medical University \\ Ningxia Hui Autonomous Region \\ Yinchuan, 750004 \\ Ningxia \\ CHINA
}

Email: wangjinbao123asd@126.com

Received: 09-11-2018

Accepted: 07-02-2019 